\title{
Laminar Shear Stress Protects Against Premature Endothelial Senescence by SIRT1-Dependent Mechanisms
}

\author{
Ji-Seok Kim PhD ${ }^{1}$, Jacqueline Sayoc BS ${ }^{2}$, Kyung-Wan Baek PhD ${ }^{1}$, Joon-Young Park PhD ${ }^{2}$ \\ ${ }^{1}$ Department of Physical Education, Gyeongsang National University, Jinju, Korea; ${ }^{2}$ Department of Kinesiology, College of Public Health \& Cardiovascular Research \\ Center, Lewis Katz School of Medicine, Temple University, Philadelphia, USA
}

\begin{abstract}
PURPOSE: Accumulation of senescent endothelial cells (ECs) and development of vascular aging have been implicated in the etiology of vascular dysfunction and disease. Aerobic exercise has been recognized as the single most effective non-pharmacological anti-aging intervention via increased laminar shear stress (LSS). This study aimed to determine the protective effects of LSS against premature senescence and the underlying mechanism.
\end{abstract}

METHODS: Carotid artery partial ligation surgery was performed on the left carotid arteries (LCAs) of C57BL/6J male mice to determine the effect of disturbed flow on the development of endothelial senescence. Senescence-associated $\beta$-galactosidase (SA- $\beta$-gal) staining was performed to measure cellular senescence. Expression levels of protein markers for cell senescence including p21, p16, and p53 were measured by western blotting.

RESULTS: En face SA- $\beta$-gal staining was identified only in the partially ligated LCAs of voluntary wheel-running mice, suggesting a direct relevance of LSS on the prevention of vascular senescence. In the in vitro $\mathrm{H}_{2} \mathrm{O}_{2}$-induced premature senescence model, preconditioning of high-flow LSS (20 dyne/ $\mathrm{cm}^{2}, 36$ hours) induced significant reduction in the percentage of SA- $\beta$-gal positive ECs. Expression of the molecular markers of cellular senescence such as p21, p16, and p53 was significantly decreased by LSS pretreatment. However, the protective effects of LSS against premature senescence were completely abolished by SIRT1 inhibition.

CONCLUSIONS: The results suggest that high-flow LSS has protective effects against oxidative stress-induced premature endothelial senescence through a SIRT1-dependent mechanism.

Key words: Laminar shear stress, Disturbed flow, SIRT1, Endothelial senescence, Vascular aging

\section{INTRODUCTION}

Vascular aging is an independent risk factor for the onset and progression of vascular diseases, such as atherosclerosis and hypertension [13]. A major mechanism of vascular aging and its pathological contribution to the functional deterioration is the accumulation of EC senescence, which is defined as an irreversible loss of cell proliferation capacity [3-5]. EC senescence can be induced by a number of factors implicated in the vascular pathologies such as oxidative stress $[1,6]$. Excessive production of reactive oxygen species generated from intracellular or extra- cellular sources accelerates the development of premature endothelial senescence [1,3] and is implicated in the pathogenesis of atherosclerosis [7]. Therefore, finding strategies for the protection of vasculatures against oxidative stress-induced premature senescence and functional impairment is believed to be a crucial step in the prevention of aging-related vascular diseases.

Aerobic exercise is known to alleviate the severity of atherosclerosis and improve vascular health [8]. The mechanism underlying these adaptive changes is believed to be governed by increased hemodynamic shear stress [9]. Aerobic exercise enhances the level of unidirectional laminar

Corresponding author: Joon-Young Park Tel +1-215-204-1957 Fax +1-215-204-4044 E-mail parkjylatemple.edu

*This work was supported by National Heart, Blood, and Lung Institute Grant (R01- HL126952) and Basic Science Research Program through the National Research

Foundation of Korea (NRF) funded by the Ministry of Education (NRF-2019R111A3A01061144).

Received 16 Apr 2021 Revised 8 May 2021 Accepted 10 May 2021 
flow and diminishes disturbed flow occurring in certain arterial geometries, such as bifurcations and curvatures [10,11]. In cultured EC, LSS is known to modulate the endothelial activation [12] by suppressing the expression of inflammatory adhesion molecules [13] and to prevent EC apoptosis, which is a known feature of atherosclerosis [14]. In spite of the accumulating evidence for the beneficial effects of LSS on the maintenance of vascular homeostasis, our understanding of the protective role of LSS against vascular senescence is largely limited.

Sirtuins, the $\mathrm{NAD}^{+}$-dependent histone deacetylases, are known to affect multiple pathways to modulate metabolic controls and to extend the life span of a wide variety of organisms [15,16]. Mammalian sirtuin 1 (SIRT1) regulates cell metabolism, the cell cycle, and apoptosis in ECs [17]. More importantly, SIRT1 has been recognized as a key regulator for the maintenance of vascular function and the prevention of endothelial senescence $[18,19]$. In a previous study, the direct contribution of enhanced LSS has been shown to cause upregulation of SIRT1 expression [20].

While multiple factors play a role in bringing about the salutary effect of aerobic exercise on the maintenance of vascular homeostasis, it was hypothesized here that the unidirectional LSS is a prominent cellular mechanism responsible for prevention against vascular senescence. Thus, the aim of this study was to determine the protective effects of LSS against the oxidative stress-induced premature endothelial senescence and to elucidate its underlying mechanism.

\section{METHODS}

\section{Animals}

Twenty C57BL/6J mice were randomly assigned to either sedentary (SED) $(n=10)$ or voluntary wheel running exercise (VW) $(n=10)$ groups. Mice in the VW group were individually housed in a rat-sized cage with a metal wheel with a diameter of $11.5 \mathrm{~cm}$ (Prevue) fitted with digital magnetic counter. Mice in the SED group were singly housed in the same rat-sized cage without the running wheel. All mice were given water and food (Purina chow) ad libitum, as previously described [21]. The animal study was carried out by procedures approved by the Temple University Institutional Animal Care and Use Committee (Permit No. 4503). All sacrifices were performed under isoflurane anesthesia.

\section{Carotid artery partial ligation}

Carotid artery partial ligation surgery was performed to determine the importance of laminar flow on the maintenance of vascular homeostasis compared to disturbed flow. The partial ligation surgergy on the left carotid arteries (LCA) of all twenty C57BL/6J male mice at 10 weeks of age. Partial ligation of LCA was carried out as previously described $[22,23]$. Briefly, anesthesia was induced by an intraperitoneal injection of a xylazine $(10 \mathrm{mg} / \mathrm{kg})$ and ketamine $(80 \mathrm{mg} / \mathrm{kg})$ mixture. the epilated area was disinfected with betadine, and a ventral midline incision (4-5 $\mathrm{mm}$ ) was made in the neck. the LCA was exposed by a blunt dissection. Three of four caudal branches of the LCA, such as the left external carotid (ECA), internal carotid (ICA), and occipital artery (OA), were surgically ligated with 6-0 silk suture leaving the superior thyroid artery (STA) as the only outflow. On the other hand, the right carotid artery (RCA) of the same mouse was left intact as the control. Then, the incision was closed with Tissue-Mend (Veterinary Product Laboratories) Mice were monitored until recovery in a chamber on a heating pad following surgery. After 1 week of recovery, ten randomly selected mice out of twenty in total were applied to VW running for a further 3 weeks.

\section{High-resolution ultrasound measurements}

All ultrasound measurements were taken using a VEVO 770 highresolution in vivo micro imaging ultrasound system with a $30-\mathrm{MHz}$ mouse probe (Visualsonics). A week after partial ligation surgeries, a high-resolution ultrasound measurement was performed. Mice were anesthetized with inhaled isoflurane, and body temperature was maintained on a heated stage for the duration of observation. Levels of anesthesia, heart rate, temperature, and respirations were continuously monitored. Pulse Wave Doppler mode was used at the midpoint of the common carotid arteries for measuring flow direction and velocity.

\section{Cell culture}

Human umbilical vein endothelial cell (HUVEC) lines from Lonza were grown in M199 medium supplemented with 20\% fetal bovine serum and endothelial cell growth supplement (Sigma-Aldrich no. E2759), and maintained at $37^{\circ} \mathrm{C}$ in a $5 \% \mathrm{CO}_{2}$ atmosphere. All experiments with HUVEC were conducted between passages 3 and 5. For shear experiments, HUVECs, grown to $90-100 \%$ confluence, were replaced with shear media and exposed to the physiological levels of shear stress for various times using a cone and plate viscometer $\left(0.5^{\circ}\right.$ cone angle), which was placed inside of a $\mathrm{CO}_{2}$ cell incubator as previously described [12, 21,24]. The shear media was M199 supplemented with 2\% fetal bovine serum and endothelial cell growth supplement. Immediately after shear 
application was over, HUVECs were treated with $100 \mu \mathrm{M} \mathrm{H}_{2} \mathrm{O}_{2}$ diluted in M199 complete culture medium for 1 hour. Then, HUVECs were trypsinized, reseeded onto gelatin-coated $100 \mathrm{~mm}$ dish, 6-well plates, 12well plates, or matrigel-coated 96-well plates according to the experimental purposes and cultured in a normal complete growth medium, containing above compounds, for a further 3 consecutive days. For the inhibition of SIRT1, HUVECs were exposed to 30 to $100 \mu \mathrm{M}$ concentrations of sirtinol (Calbiochem no. 566320), which is a cell permeable 2-hydroxy1-napthaldehyde derivative and a chemical inhibitor of $\mathrm{NAD}^{+}$-dependent protein deacetylases of sirtuins.

\section{Senescence-associated $\beta$-galactosidase staining}

The proportion of Senescence-Associated $\beta$-Galactosidase (SA- $\beta$-gal) positive cells in cultured HUVECs or carotid arteries isolated from C57BL/6 mice were determined as described previously [25]. Briefly, cultured cells or vessel tissues were fixed in a fixative solution containing $2 \%$ formaldehyde and $0.2 \%$ glutaraldehyde for 15 minutes after washing with PBS. Cells or vessel tissues were incubated overnight at $37^{\circ} \mathrm{C}$ in a dry incubator in a $\beta$-Galactosidase staining solution, which contains 1 mg/mL X-gal in N-N-dimethylformamide (DMF), 40 mM citric acid/ sodium phosphate ( $\mathrm{pH}$ 6.0), $150 \mathrm{mM} \mathrm{NaCl}, 2 \mathrm{mM} \mathrm{MgCl}_{2}, 5 \mathrm{mM}$ potassium ferrocyanide, and $5 \mathrm{mM}$ potassium ferricyanide. The proportion of SA- $\beta$-gal positive cells was determined by counting the number of blue-stained cells out of the total number of cells in the same field of 200 $\times$ total magnification (Axiovert 40 CFL, Zeiss).

\section{Western blotting and antibodies}

Antibodies were from the following sources: rabbit monoclonal antiSIRT1 (Cell signaling no. 2496), rabbit monoclonal anti-p21 (Cell signaling no. 2947), mouse monoclonal anti-p53 (Santa Cruz no. sc-126), and rabbit monoclonal anti-p16 (Abcam no. ab51243) antibodies. Mouse monoclonal anti- $\alpha$-Tubulin (Sigma no. T9026) and mouse monoclonal anti- $\beta$-Actin (Sigma no. A1978) were used as the internal controls.

ECs were homogenized in ice-cold RIPA lysis buffer with protease inhibitor (Roche no. 11836153001). Proteins from cell lysates were resolved by Tris-glycine SDS PAGE and transferred to Immobilon-P PVDF membrane for standard ECL Western blotting. Total protein expression was detected by chemiluminescence. Band densitometry analyses were completed using Image J software (National Institute of Health), to direct comparisons between experimental sets. The values calculated by Image J were arbitrary units. The densities of the selected protein bands were expressed relative to the densities of the internal control genes.

\section{Statistical analysis}

Data are presented as mean \pm SEM. Differences across experimental conditions were assessed by analysis of variance followed by post-hoc testing with the Fisher's least significant difference test. $p<.05$ was considered statistically significant for all analyses.

\section{RESULTS}

\section{Partial ligation-induced disturbed flow and vascular senescence}

To investigate the negative effects of disturbed flow on in vivo vascular senescence compared to the positive effects of laminar flow for the maintenance of vascular homeostasis, three of four caudal branches of the LCA, such as the ECA, ICA, and OA, were surgically ligated leaving the STA as the only outflow. On the other hand, the RCA of the same mouse was left intact as the control. Flow velocity and direction of the LCA and RCA were determined by a high-resolution in vivo micro imaging ultrasound system. Results of the ultrasound showed the partial ligation-induced flow reversal in the ranges of 350 to $500 \mathrm{~mm} / \mathrm{s}$ with reduced velocity to the unidirectional flow in the LCA (Fig. 1B). After 3 weeks of VW or SED after partial ligation surgery and a week recovery, mice were sacrificed and their carotid arteries were isolated for SA- $\beta$-gal en-face staining analysis. SA- $\beta$-gal staining was identified only at the partially-ligated LCA of VW mice, while the LCA of SED mice and RCA from both SED and VW mice were not stained. This result represents the partial ligation and VW running-induced promoted disturbed flow in LCA leads to the vascular senescence, while laminar flow keeps the RCA intact (Fig. 1A). The average running distance of LCA partial ligated VW mice was $5.51 \mathrm{~km} /$ day and the body weight was significantly different in between groups and time points (pre-SED $25.39 \pm 0.72 \mathrm{~g}$, post-SED $25.83 \pm 0.61 \mathrm{~g}$, pre-VW $25.54 \pm 0.40 \mathrm{~g}$, post-VW $25.96 \pm 0.33 \mathrm{~g}$ ) demonstrating their behaviors, such as activity and food intake, were not affected by the ligation surgery.

\section{SIRT1 and oxidative stress-induced premature endothelial senescence}

To investigate the role of SIRT1 in the regulation of EC senescence, SIRT1 was inhibited by the treatment of sirtinol, a specific chemical inhibitor of sirtuins, for 24 hours in concentrations of 30,60 , and $100 \mu \mathrm{M}$. 


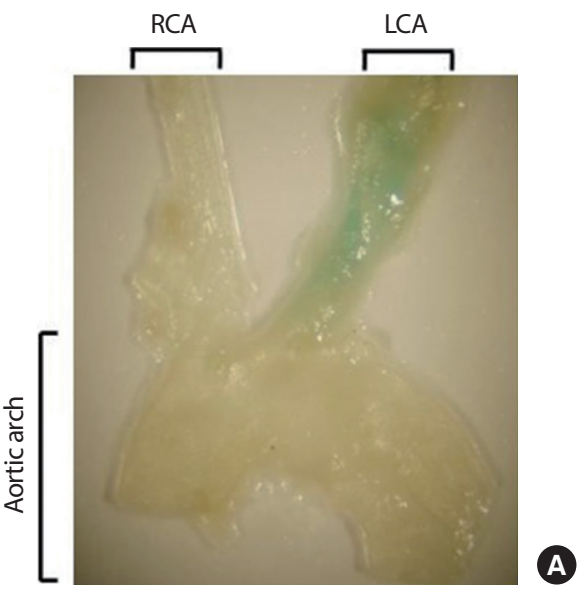

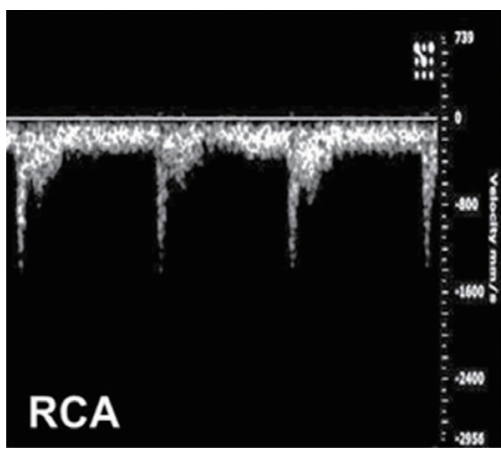

Velocity $(\mathrm{mm} / \mathrm{s})$

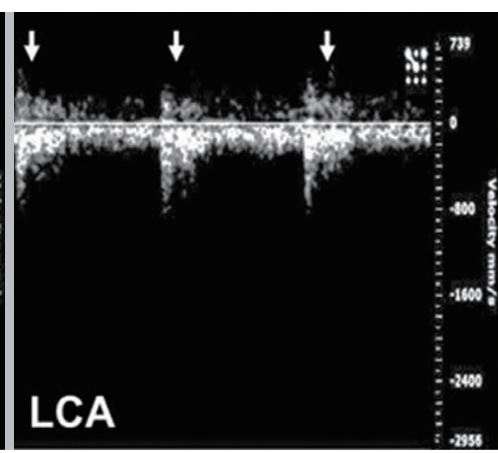

Velocity $(\mathrm{mm} / \mathrm{s})$

Fig. 1. Partial ligation-induced promoted disturbed flow accelerates vascular senescence. Senescence-associated $\beta$-galactosidase (SA- $\beta$-gal) en-face staining analysis in mouse coronary arteries after 4 weeks of LCA partial-ligation surgery and voluntary running (A). Echocardiography analysis 1-week after LCA partial-ligation surgery (B). Arrows indicate flow reversal. LCA, left carotid artery; RCA, right carotid artery; ECA, external carotid artery; ICA, internal carotid artery; OA, occipital artery; STA, superior thyroid artery.

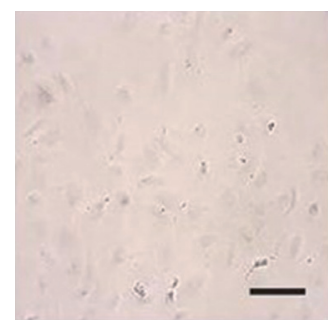

0

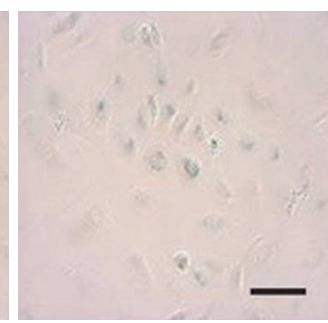

30

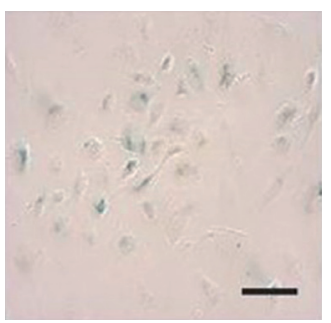

60

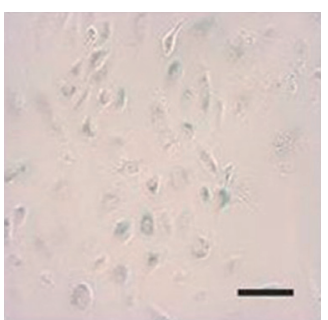

100
A
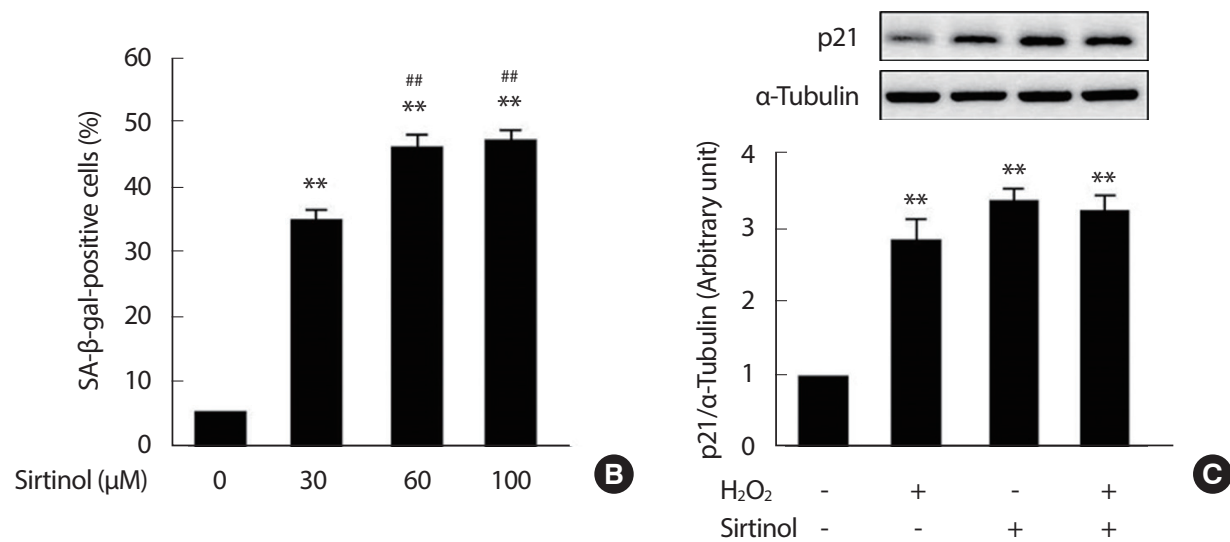

Fig. 2. Depletion of SIRT1 induces endothelial senescence. Representative SA- $\beta$-gal staining pictures (A). The proportion of SA- $\beta$-gal-positive cells (B). p21 expression analyzed by Western blotting (C). HUVECs were treated with sirtinol in concentrations of 30, 60, and $100 \mu \mathrm{M}$ for 24 hours or $100 \mu \mathrm{M} \mathrm{H} \mathrm{H}_{2} \mathrm{O}_{2}$ for 1 hour. Then, the cells were reseeded and cultured in normal complete medium for a further 3 consecutive days. The proportion of SA- $\beta$-gal positive cells was determined by counting the number of stained cells out of the total number of cells in the same field. Cells were counted in 60 independent fields. Scale bar $=100 \mu \mathrm{m} .{ }^{* *} p<.01$ compared to non-treated control; ${ }^{\# \#} p<.01$ compared to $30 \mu \mathrm{M}$ sirtinol treatment.

Then, sirtinol-treated HUVECs were cultured in complete growth media for 3 consecutive days. The proportion of SA- $\beta$-gal-positive cells was dramatically increased in sirtinol-treated ECs in a dose-dependent manner (Fig. 2A, B). More than 45\% of ECs treated with sirtinol (60 $\mu \mathrm{M}$ or
$100 \mu \mathrm{M})$ were SA- $\beta$-gal-positive, while only about $6 \%$ of vehicle-treated ECs were SA- $\beta$-gal-positive. Senescent ECs also exhibited morphological changes of enlarged and flattened shapes. Likewise, the expressions of p21, a molecular marker of cell senescence, were significantly increased 


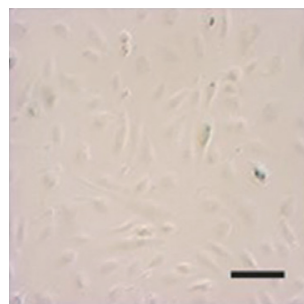

NT

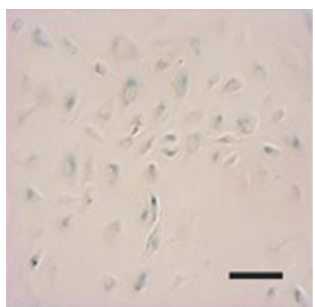

$\mathrm{H}_{2} \mathrm{O}_{2}$

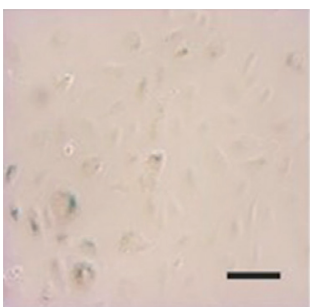

$\mathrm{LSS}+\mathrm{H}_{2} \mathrm{O}_{2}$

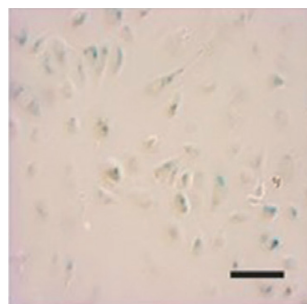

LSS + Sirtinol $+\mathrm{H}_{2} \mathrm{O}_{2}$
A

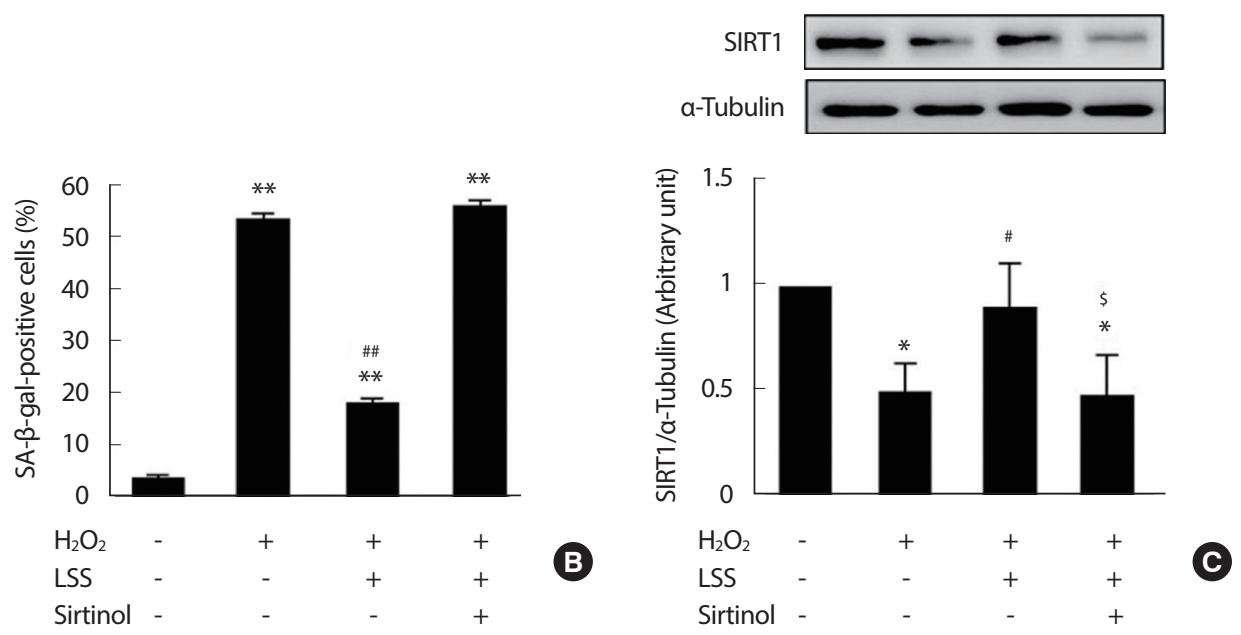

Fig. 3. LSS inhibits oxidative stress-induced premature endothelial senescence through the SIRT1 pathway. Representative SA- $\beta$-gal staining pictures (A). The proportion of SA- $\beta$-gal-positive staining cells (B). SIRT1 expression analyzed by Western blotting. The proportion of SA- $\beta$-gal positive cells was determined by counting the number of stained cells out of the total number of cells in the same field. Cells were counted in 90 independent fields. Scale bar $=100 \mu \mathrm{m}$. NT=Non-treated control; ${ }^{*} p<.05$ and ${ }^{* *} p<.01$ compared to NT; ${ }^{*} p<.05$ and ${ }^{\# \#} p<.01$ compared to $\mathrm{H}_{2} \mathrm{O}_{2}$ treatment; ${ }^{5} p<.05$ compared to $\mathrm{H}_{2} \mathrm{O}_{2}$ and LSS co-treatment.

in sirtinol and/or $\mathrm{H}_{2} \mathrm{O}_{2}$-treated ECs (Fig. 2C). This result confirms that the role of SIRT1 in the regulation of oxidative stress-induced premature endothelial senescence.

\section{Protective effects of LSS on endothelial senescence in SIRT-dependent mechanism}

To determine the protective effects of LSS on $\mathrm{H}_{2} \mathrm{O}_{2}$-induced premature endothelial senescence, HUVECs were pre-treated with LSS in the intensity of 20 dyne $/ \mathrm{cm}^{2}$ for 12 hours followed by 5 dyne $/ \mathrm{cm}^{2}$ LSS for 24 hours before the treating $\mathrm{H}_{2} \mathrm{O}_{2}$. After 3 consecutive days of aging in complete growth medium, more than $50 \%$ of $\mathrm{H}_{2} \mathrm{O}_{2}$-treated ECs were SA- $\beta$-gal-positive, while only about $4 \%$ of vehicle-treated ECs were SA- $\beta$ -gal-positive. This result was partly reversed in LSS pre-treated ECs. In LSS pre-conditioned HUVECs, only $18 \%$ of ECs were SA- $\beta$-gal-positive. However, the effect of LSS on the alleviation of EC senescence was completely abolished (55\% SA- $\beta$-gal-positive) when SIRT1 was inhibited by sirtinol treatment (Fig. 3). The expressions of p21, p16, and p53, which are the molecular markers of cellular senescence, were in accordance with the results of SA- $\beta$-gal staining (Fig. 4). In HUVECs that underwent $\mathrm{H}_{2} \mathrm{O}_{2}$-induced premature senescence, $\mathrm{p} 21$ expression was increased by three-fold, p16 by about four-fold, and p53 by two-fold after 3 consecutive days of aging. However, the expression levels were decreased to basal level in LSS pre-conditioned ECs, while the effect of LSS disappeared in the SIRT1-inhibited condition. These results represent that LSS can partly prevent $\mathrm{H}_{2} \mathrm{O}_{2}$-induced premature endothelial senescence through SIRT1-dependent mechanism.

\section{DISCUSSION}

Endothelial senescence, an irreversible loss of cell proliferation capacity, is directly related to the functional deterioration of ECs and age-associated diseases, including hypertension and atherosclerosis [1,5,31]. The present results demonstrated that LSS provides a prominent cellular mechanism responsible for the prevention of oxidative stress-induced premature senescence in a SIRT1-dependent mechanism in vascular ECs.

We first demonstrated in the present study that the promoted dis- 

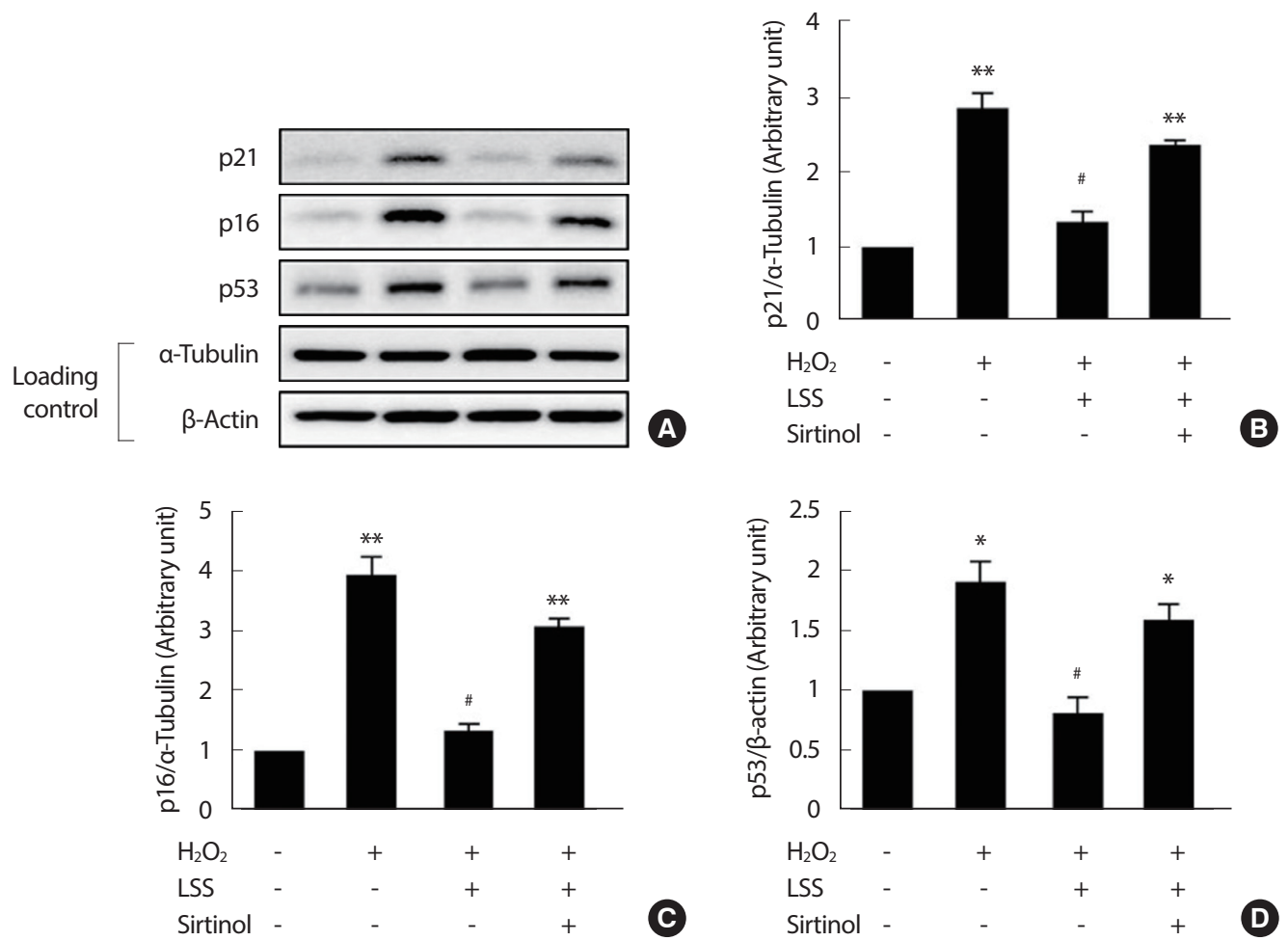

Fig. 4. LSS decreases the expressions of molecular markers of cellular senescence. p21, p16, and p53 expressions were analyzed by Western blotting (A-D). Bar graphs are results of densitometry analyses. Each column represents mean \pm SEM from more than 3 independent experiments. Scale bar $=100 \mu m$. NT, non-treated control; LSS, laminar shear stress. ${ }^{*} p<.05$, ${ }^{* *} p<.01$ compared to NT; ${ }^{*} p<.05$, ${ }^{\# \#} p<.01$ compared to $\mathrm{H}_{2} \mathrm{O}_{2}$ treatment.

turbed flow (or flow-reversal) induced by the carotid artery partial ligation surgery combined with VW running resulted in vascular senescence, while laminar flow keeps the coronary arteries intact in the same mice (Fig. 1). This result is attributed to the repeated exposure of endothelium to enhanced flow-reversal with attenuated laminar flow. This finding is consistent with the previous studies demonstrating that the partial ligation-induced disturbed flow leads to the endothelial dysfunction and development of atherosclerosis in the carotid arteries of highfat diet treated $\mathrm{ApoE}^{-/-}$mice $[22,23]$. A recent study from another group has also shown that disturbed flow promotes endothelial senescence in the aortic arch of high-fat diet treated $\operatorname{LDLR}^{-/}$mice [27]. Our current study shows that SA- $\beta$-gal staining was identified only at the partially-ligated VW mice's LCA, while the LCA of SED mice and RCAs from both SED and VW mice were not stained. These results represent that disturbed flow induced by the partial ligation combined with voluntary running can directly induce vascular senescence. Importantly, this is the first finding which demonstrated that vascular wall shear stress itself is a crucial determinant for the regulation of vascular senescence in wild type mice.

Diverse pharmacological treatments on vascular ECs and the trials to determine their underlying mechanisms, such as eNOS and SIRT1 activation-dependent pathways, on the prevention against EC senescence and dysfunction have been studied extensively [19,28-30]. Our data, presented here, add a new methodological insight into the prevention of EC senescence and dysfunction. This study shows that unidirectional LSS is a direct and prominent cellular mechanism responsible for the protection against oxidative stress-induced premature EC senescence. In HUVECs that underwent oxidative stress-induced premature senescence, the rate of SA- $\beta$-gal-positive cells (Fig. 3) and the expressions of p53, p21, and p16 were significantly increased (Fig. 4). Interestingly, these results were reversed by LSS pre-treatment. However, these protective effects of LSS on endothelial senescence were completely abolished by SIRT1 inhibition. These results represent that LSS pre-conditioning has protective effects against oxidative stress-induced premature endothelial senescence through a SIRT1-dependent mechanism.

Upregulation of SIRT1 has been deeply implicated in LSS. For instance, enhanced LSS increased endothelial function via SIRT1-dependent pathway in cultured ECs [20]. Furthermore, it was shown in our previous study that the increased LSS attenuates EMP production, a determinant of endothelial activation, via SIRT1 enhancement and mito- 
chondrial biogenesis both in in vitro and in vivo studies [12]. Also, previous studies have shown that the treatment of a known SIRT1 activator, resveratrol, in cultured human vascular ECs increases the oxidativestress resistance by scavenging $\mathrm{H}_{2} \mathrm{O}_{2}$ and preventing oxidative stress-induced cell death $[33,34]$. The present study has shown the possibility of protective effects of LSS on the oxidative stress-induced premature endothelial senescence.

In another previous study, it was determined that SIRT1 inhibition induces premature senescence-like phenotypes in human vascular ECs [28]. SIRT1 inhibition by the treatment of sirtinol or siRNA induced cell growth arrest and increased the proportion of SA- $\beta$-gal-positive cells. Conversely, SIRT1 overexpression prevented oxidative stress-induced SA- $\beta$-gal activity in human ECs, suggesting that SIRT1 exerts protective effects against premature endothelial senescence [28]. In this regard, we also confirmed the role of SIRT1 in premature endothelial senescence. SIRT1 inhibition by the treatment of sirtinol increased the proportion of SA- $\beta$-gal-positive cells as well as the levels of p21 expressions, representing that the depletion of SIRT1 itself can be a direct contributor for endothelial senescence (Fig. 2).

\section{CONCLUSION}

The reversal of vascular aging has been a critical issue for the prevention of cardiovascular diseases. SIRT1 has been proposed as a key contributor for the inhibition of cellular senescence. The present study highlights the role of LSS in the protection against oxidative stress-induced premature endothelial senescence in the SIRT1-dependent mechanisms. Moreover, it was confirmed in our in vivo carotid partial ligation models that vascular wall shear stress itself is a crucial determinant for the regulation of vascular senescence. Finally, it is suggested that aerobic exercise can be a potentially effective strategy for the maintenance of vascular homeostasis against oxidative stress-induced premature vascular aging.

\section{CONFLICT OF INTEREST}

The authors declare that they do not have any conflicts of interest.

\section{AUTHOR CONTRIBUTIONS}

Conceptualization: J Kim, J Park; Data curation: J Kim; Formal analysis: J Kim; Funding acquisition: J Park; Methodology: J Kim; Project ad- ministration: J Park; Visualization: J Sayoc, K Baek; Writing-original draft: J Kim; Writing-review \& editing: J Park, J Sayoc, K Baek.

\section{ORCID}

Ji-Seok Kim

Kyung-Wan Baek

Joon-Young Park https:/orcid.org/0000-0002-3023-1999

https://orcid.org/0000-0002-8445-3773

https://orcid.org/0000-00002-7705-7086

\section{REFERENCES}

1. Erusalimsky JD. Vascular endothelial senescence: from mechanisms to pathophysiology. J Appl Physiol (1985). 2009;106(1):326-32.

2. Lakatta EG. Arterial and cardiac aging: major shareholders in cardiovascular disease enterprises: Part III: cellular and molecular clues to heart and arterial aging. Circulation. 2003;107(3):490-7.

3. Minamino T, Miyauchi H, Yoshida T, Ishida Y, Yoshida H, et al. Endothelial cell senescence in human atherosclerosis: role of telomere in endothelial dysfunction. Circulation. 2002;105(13):1541-4.

4. Erusalimsky JD, Kurz DJ. Cellular senescence in vivo: its relevance in ageing and cardiovascular disease. Exp Gerontol. 2005;40(8-9):634-42.

5. Wang JC, Bennett M. Aging and atherosclerosis: mechanisms, functional consequences, and potential therapeutics for cellular senescence. Circ Res. 2012;111(2):245-59.

6. Marin C, Yubero-Serrano EM, Lopez-Miranda J, Perez-Jimenez F. Endothelial aging associated with oxidative stress can be modulated by a healthy mediterranean diet. Int J Mol Sci. 2013;14(5):8869-89.

7. Liu H, Colavitti R, Rovira, II, Finkel T. Redox-dependent transcriptional regulation. Circ Res. 2005;97(10):967-74.

8. Okabe TA, Shimada K, Hattori M, Murayama T, Yokode M, et al. Swimming reduces the severity of atherosclerosis in apolipoprotein E deficient mice by antioxidant effects. Cardiovasc Res. 2007;74(3):53745 .

9. Malek AM, Alper SL, Izumo S. Hemodynamic shear stress and its role in atherosclerosis. JAMA. 1999;282(21):2035-42.

10. Taylor CA, Hughes TJ, Zarins CK. Effect of exercise on hemodynamic conditions in the abdominal aorta. J Vasc Surg. 1999;29(6):1077-89.

11. Cheng C. Abdominal aortic hemodynamic conditions in healthy subjects aged 50-70 at rest and during lower limb exercise: in vivo quantification using MRI. Atherosclerosis. 2003;168(2):323-31.

12. Kim JS, Kim B, Lee H, Thakkar S, Babbitt DM, et al. Shear stress-in- 
duced mitochondrial biogenesis decreases the release of microparticles from endothelial cells. Am J Physiol Heart Circ Physiol. 2015;309(3): H425-33.

13. Ando J, Tsuboi H, Korenaga R, Takada Y, Toyama-Sorimachi N, et al. Shear stress inhibits adhesion of cultured mouse endothelial cells to lymphocytes by downregulating VCAM-1 expression. Am J Physiol. 1994;267(3 Pt 1):C679-87.

14. Dimmeler S, Haendeler J, Rippmann V, Nehls M, Zeiher AM. Shear stress inhibits apoptosis of human endothelial cells. FEBS Lett. 1996; 399(1-2):71-4.

15. Haigis MC, Sinclair DA. Mammalian sirtuins: biological insights and disease relevance. Annu Rev Pathol. 2010;5:253-95.

16. Braunstein M, Rose AB, Holmes SG, Allis CD, Broach JR. Transcriptional silencing in yeast is associated with reduced nucleosome acetylation. Genes Dev. 1993;7(4):592-604.

17. Borradaile NM, Pickering JG. NAD(+), sirtuins, and cardiovascular disease. Curr Pharm Des. 2009;15(1):110-7.

18. Potente M, Ghaeni L, Baldessari D, Mostoslavsky R, Rossig L, et al. SIRT1 controls endothelial angiogenic functions during vascular growth. Genes Dev. 2007;21(20):2644-58.

19. Ota H, Eto M, Kano MR, Kahyo T, Setou M, et al. Induction of endothelial nitric oxide synthase, SIRT1, and catalase by statins inhibits endothelial senescence through the Akt pathway. Arterioscler Thromb Vasc Biol. 2010;30(11):2205-11.

20. Chen Z, Peng IC, Cui X, Li YS, Chien S, et al. Shear stress, SIRT1, and vascular homeostasis. Proc Natl Acad Sci U S A. 2010;107(22):1026873.

21. Kim B, Lee H, Kawata K, Park JY. Exercise-mediated wall shear stress increases mitochondrial biogenesis in vascular endothelium. PLoS One. 2014;9(11):e111409.

22. Nam D, Ni CW, Rezvan A, Suo J, Budzyn K, et al. Partial carotid ligation is a model of acutely induced disturbed flow, leading to rapid endothelial dysfunction and atherosclerosis. Am J Physiol Heart Circ Physiol. 2009;297(4):H1535-43.

23. Nam D, Ni CW, Rezvan A, Suo J, Budzyn K, et al. A model of disturbed flow-induced atherosclerosis in mouse carotid artery by partial ligation and a simple method of RNA isolation from carotid endothelium. J Vis Exp. 2010. doi:10.3791/1861(40).
24. Park JY, Farrance IK, Fenty NM, Hagberg JM, Roth SM, et al. NFKB1 promoter variation implicates shear-induced NOS3 gene expression and endothelial function in prehypertensives and stage I hypertensives. Am J Physiol Heart Circ Physiol. 2007;293(4):H2320-7.

25. Dimri GP, Lee X, Basile G, Acosta M, Scott G, et al. A biomarker that identifies senescent human cells in culture and in aging skin in vivo. Proc Natl Acad Sci U S A. 1995;92(20):9363-7.

26. Brown MD, Feairheller DL, Thakkar S, Veerabhadrappa P, Park JY. Racial differences in tumor necrosis factor-alpha-induced endothelial microparticles and interleukin-6 production. Vasc Health Risk Manag. 2011;7:541-50.

27. Warboys CM, de Luca A, Amini N, Luong L, Duckles H, et al. Disturbed Flow Promotes Endothelial Senescence via a p53-Dependent Pathway. Arterioscler Thromb Vasc Biol 2014. doi:10.1161/ATVBAHA.114.303415.

28. Ota H, Akishita M, Eto M, Iijima K, Kaneki M, et al. Sirtl modulates premature senescence-like phenotype in human endothelial cells. J Mol Cell Cardiol. 2007;43(5):571-9.

29. Kao CL, Chen LK, Chang YL, Yung MC, Hsu CC, et al. Resveratrol protects human endothelium from $\mathrm{H}_{2} \mathrm{O}_{2}$-induced oxidative stress and senescence via SirT1 activation. J Atheroscler Thromb. 2010;17(9):970-9.

30. Botden IP, Oeseburg H, Durik M, Leijten FP, Van Vark-Van Der Zee LC, et al. Red wine extract protects against oxidative-stress-induced endothelial senescence. Clin Sci (Lond). 2012;123(8):499-507.

31. Feletou M, Vanhoutte PM. Endothelial dysfunction: a multifaceted disorder (The Wiggers Award Lecture). Am J Physiol Heart Circ Physiol. 2006;291(3):H985-1002.

32. Lin JR, Shen WL, Yan C, Gao PJ. Downregulation of dynamin-related protein 1 contributes to impaired autophagic flux and angiogenic function in senescent endothelial cells. Arterioscler Thromb Vasc Biol. 2015;35(6):1413-22.

33. Ungvari Z, Orosz Z, Rivera A, Labinskyy N, Xiangmin Z, et al. Resveratrol increases vascular oxidative stress resistance. Am J Physiol Heart Circ Physiol. 2007;292(5):H2417-24.

34. Ungvari Z, Bagi Z, Feher A, Recchia FA, Sonntag WE, et al. Resveratrol confers endothelial protection via activation of the antioxidant transcription factor Nrf2. Am J Physiol Heart Circ Physiol. 2010;299 (1):H18-24. 